\title{
T-Convolution and its applications to $n$-dimensional distributions
}

\author{
A. A. Pogorui, D. O. Kovalenko and Ramón M. Rodríguez-Dagnino \\ Communicated by A. F. Turbin
}

\begin{abstract}
In this paper we introduce the notion of T-convolution, which is a generalization of convolution to higher dimensions. By using T-convolution we construct $n$-dimensional distributions having $n+1$ axes of symmetry. In addition, we can generalize well-known symmetric probability distributions in one dimension to higher dimensions. In particular, we consider generalizations of Laplace and triangle continuous distributions and we show their plots in the two-dimensional case. As an example of discrete distributions, we study the T-convolution of Poisson distributions in the plane.
\end{abstract}

Key words. Probability; convolutions; multivariate pdf.

AMS classification. 47N30, 65C50.

\section{Introduction}

As usual, the distribution of a random $n$-dimensional vector is determined through its distribution on $n$-dimensional rectangular parallelepipeds. In this work we consider $n$-dimensional distributions of random vectors through their distribution in polyhedrons, which are distinct from parallelepipeds. For such a purpose we introduce the so-called T-convolution which is a generalization of the convolution of two functions. For $n$-dimensional distributions we consider polyhedrons which can pack the $n$-dimensional space $\mathbb{R}^{n}$.

\section{T-Convolution on a plane}

Let $\mathbb{V}^{2}$ be the two-dimensional vector space. Denote by $\tau_{1}, \tau_{2}, \tau_{3}$ three vectors belonging to $\mathbb{V}^{2}$. Let us assume that these vectors have their origin in the center of a regular or equilateral triangle and terminal points in the vertices. Let us also denote by $\xi_{1}, \xi_{2}, \xi_{3}$ three independent random variables with corresponding probability density functions (pdf) $f_{\xi_{1}}(x), f_{\xi_{2}}(x), f_{\xi_{3}}(x)$. 
Consider the random vector $\boldsymbol{\xi}=\xi_{1} \boldsymbol{\tau}_{1}+\xi_{2} \boldsymbol{\tau}_{2}+\xi_{3} \boldsymbol{\tau}_{3}$. The pdf of this vector in the set $\left\{x_{1} \boldsymbol{\tau}_{1}+x_{2} \boldsymbol{\tau}_{2} ; x_{1}, x_{2} \geq 0\right\}$ is determined as follows:

$$
\begin{aligned}
& P\left\{\boldsymbol{\xi} \in\left(d x_{1} \boldsymbol{\tau}_{1}+d x_{2} \boldsymbol{\tau}_{2}\right)\right\} \\
& =P\left\{\xi_{1} \boldsymbol{\tau}_{1}+\xi_{2} \boldsymbol{\tau}_{2}+\xi_{3} \boldsymbol{\tau}_{3} \in\left(d x_{1} \boldsymbol{\tau}_{1}+d x_{2} \boldsymbol{\tau}_{2}\right)\right\} \\
& =P\left\{\left(\xi_{1}-\xi_{3}\right) \boldsymbol{\tau}_{1}+\left(\xi_{2}-\xi_{3}\right) \boldsymbol{\tau}_{2} \in\left(d x_{1} \boldsymbol{\tau}_{1}+d x_{2} \boldsymbol{\tau}_{2}\right)\right\} \\
& =\int_{-\infty}^{\infty} P\left\{\left(\xi_{1}-u\right) \in d x_{1},\left(\xi_{2}-u\right) \in d x_{2} \mid \xi_{3}=u\right\} P\left\{\xi_{3} \in d u\right\} \\
& =\left(\int_{-\infty}^{\infty} f_{\xi_{1}}\left(x_{1}+u\right) f_{\xi_{2}}\left(x_{2}+u\right) f_{\xi_{3}}(u) d u\right) d x_{1} d x_{2}
\end{aligned}
$$

Now, denote by $f_{\boldsymbol{\xi}}\left(x_{1}, x_{2}\right)$ the pdf of the random vector $\boldsymbol{\xi}$. It is easy to see that

$$
\begin{aligned}
P\left\{\boldsymbol{\xi} \in\left(d x_{1} \boldsymbol{\tau}_{1}+d x_{2} \boldsymbol{\tau}_{2}\right)\right\} & =f_{\boldsymbol{\xi}}\left(x_{1}, x_{2}\right) d x_{1} d x_{2} \sin \left(\boldsymbol{\tau}_{1}, \boldsymbol{\tau}_{2}\right) \\
& =f_{\boldsymbol{\xi}}\left(x_{1}, x_{2}\right) d x_{1} d x_{2} \sqrt{3} / 2
\end{aligned}
$$

Hence,

$$
f_{\boldsymbol{\xi}}\left(x_{1}, x_{2}\right)=\frac{2}{\sqrt{3}} \int_{-\infty}^{\infty} f_{\xi_{1}}\left(x_{1}+u\right) f_{\xi_{2}}\left(x_{2}+u\right) f_{\xi_{3}}(u) d u
$$

Similarly, for the set $\left\{\tau_{1} x_{1}+\tau_{3} x_{3} ; x_{1}, x_{3} \geq 0\right\}$ the pdf is given by

$$
f_{\boldsymbol{\xi}}\left(x_{1}, x_{3}\right)=\frac{2}{\sqrt{3}} \int_{-\infty}^{\infty} f_{\xi_{1}}\left(x_{1}+u\right) f_{\xi_{3}}\left(x_{3}+u\right) f_{\xi_{2}}(u) d u
$$

and for the set $\left\{\tau_{2} x_{2}+\tau_{3} x_{3} ; x_{2}, x_{3} \geq 0\right\}$ we have

$$
f_{\boldsymbol{\xi}}\left(x_{2}, x_{3}\right)=\frac{2}{\sqrt{3}} \int_{-\infty}^{\infty} f_{\xi_{2}}\left(x_{2}+u\right) f_{\xi_{3}}\left(x_{3}+u\right) f_{\xi_{1}}(u) d u .
$$

Now, let us denote by $\mathrm{H}_{2}(t)$ a regular hexagon where $t>0$ is the distance from the center to the vertices. The probability distribution of a random vector $\xi$ in this hexagon can be written as

$$
\begin{aligned}
P\left\{\boldsymbol{\xi} \in \mathrm{H}_{2}(t)\right\}= & \frac{2}{\sqrt{3}} \int_{0}^{t} \int_{0}^{t} \int_{-\infty}^{\infty} f_{\xi_{1}}\left(x_{1}+u\right) f_{\xi_{2}}\left(x_{2}+u\right) f_{\xi_{3}}(u) d u d x_{1} d x_{2} \\
& +\frac{2}{\sqrt{3}} \int_{0}^{t} \int_{0}^{t} \int_{-\infty}^{\infty} f_{\xi_{2}}\left(x_{2}+u\right) f_{\xi_{3}}\left(x_{3}+u\right) f_{\xi_{1}}(u) d u d x_{2} d x_{3} \\
& +\frac{2}{\sqrt{3}} \int_{0}^{t} \int_{0}^{t} \int_{-\infty}^{\infty} f_{\xi_{1}}\left(x_{1}+u\right) f_{\xi_{3}}\left(x_{3}+u\right) f_{\xi_{2}}(u) d u d x_{1} d x_{3} .
\end{aligned}
$$


Definition 2.1. We define as the two-dimensional T-convolution the following operator of the three pdf $f_{\xi_{1}}(x), f_{\xi_{2}}(x), f_{\xi_{3}}(x)$ :

$$
\begin{aligned}
& f_{\xi_{1}} \circledast f_{\xi_{2}} \circledast f_{\xi_{3}}\left(x_{1}, x_{2}, x_{3}\right) \\
& :=\left\{\begin{array}{lll}
\frac{2}{\sqrt{3}} \int_{-\infty}^{\infty} f_{\xi_{1}}\left(x_{1}+u\right) f_{\xi_{2}}\left(x_{2}+u\right) f_{\xi_{3}}(u) d u & \text { if } & x_{1}>0, x_{2} \geq 0, \\
\frac{2}{\sqrt{3}} \int_{-\infty}^{\infty} f_{\xi_{2}}\left(x_{2}+u\right) f_{\xi_{3}}\left(x_{3}+u\right) f_{\xi_{1}}(u) d u & \text { if } & x_{2}>0, x_{3} \geq 0, \\
\frac{2}{\sqrt{3}} \int_{-\infty}^{\infty} f_{\xi_{3}}\left(x_{3}+u\right) f_{\xi_{1}}\left(x_{1}+u\right) f_{\xi_{2}}(u) d u & \text { if } & x_{1} \geq 0, x_{3}>0 .
\end{array}\right.
\end{aligned}
$$

Now, let us consider three independent discrete random variables $\eta_{1}, \eta_{2}, \eta_{3}$ with probability mass functions (pmf) $p_{\eta_{1}}(k), p_{\eta_{2}}(k), p_{\eta_{3}}(k), k \in \mathbb{N}_{0}=\{0,1,2, \ldots, \infty\}$, respectively. The probability distribution of the random vector $\boldsymbol{\eta}=\eta_{1} \boldsymbol{\tau}_{1}+\eta_{2} \boldsymbol{\tau}_{2}+\eta_{3} \boldsymbol{\tau}_{3}$ on the lattice $\left\{m_{1} \boldsymbol{\tau}_{1}+m_{2} \boldsymbol{\tau}_{2} ; m_{1}, m_{2} \in \mathbb{N}_{0}\right\}$ is of the form

$$
\begin{aligned}
& P\left\{\boldsymbol{\eta}=\left(m_{1} \boldsymbol{\tau}_{1}+m_{2} \boldsymbol{\tau}_{2}\right)\right\} \\
& =\sum_{k=0}^{\infty} P\left\{\left(\eta_{1}-k\right) \boldsymbol{\tau}_{1}+\left(\eta_{2}-k\right) \boldsymbol{\tau}_{2}=m_{1} \boldsymbol{\tau}_{1}+m_{2} \boldsymbol{\tau}_{2} \mid \eta_{3}=k\right\} P\left\{\eta_{3}=k\right\} \\
& =\sum_{k=0}^{\infty} P\left\{\eta_{1}=m_{1}+k\right\} P\left\{\eta_{2}=m_{2}+k\right\} P\left\{\eta_{3}=k\right\} \\
& =\sum_{k=0}^{\infty} p_{\eta_{1}}\left(m_{1}+k\right) p_{\eta_{2}}\left(m_{2}+k\right) p_{\eta_{3}}(k) .
\end{aligned}
$$

Similarly, for the lattice $\left\{m_{1} \boldsymbol{\tau}_{1}+m_{3} \tau_{3} ; m_{1}, m_{3} \in \mathbb{N}_{0}\right\}$ we can write it as

$$
\begin{aligned}
& P\left\{\boldsymbol{\eta}=\left(m_{1} \boldsymbol{\tau}_{1}+m_{3} \boldsymbol{\tau}_{3}\right)\right\} \\
& =\sum_{k=0}^{\infty} P\left\{\left(\eta_{1}-k\right) \boldsymbol{\tau}_{1}+\left(\eta_{3}-k\right) \boldsymbol{\tau}_{3}=m_{1} \boldsymbol{\tau}_{1}+m_{3} \boldsymbol{\tau}_{3} \mid \eta_{2}=k\right\} P\left\{\eta_{2}=k\right\} \\
& =\sum_{k=0}^{\infty} P\left\{\eta_{1}=m_{1}+k\right\} P\left\{\eta_{3}=m_{3}+k\right\} P\left\{\eta_{2}=k\right\} \\
& =\sum_{k=0}^{\infty} p_{\eta_{1}}\left(m_{1}+k\right) p_{\eta_{3}}\left(m_{3}+k\right) p_{\eta_{2}}(k)
\end{aligned}
$$

and for the lattice $\left\{m_{2} \boldsymbol{\tau}_{2}+m_{3} \boldsymbol{\tau}_{3} ; m_{2}, m_{3} \in \mathbb{N}_{0}\right\}$ as

$$
\begin{aligned}
& P\left\{\boldsymbol{\eta}=\left(m_{2} \boldsymbol{\tau}_{2}+m_{3} \boldsymbol{\tau}_{3}\right)\right\} \\
& =\sum_{k=0}^{\infty} P\left\{\left(\eta_{2}-k\right) \boldsymbol{\tau}_{2}+\left(\eta_{3}-k\right) \boldsymbol{\tau}_{3}=m_{2} \boldsymbol{\tau}_{2}+m_{3} \boldsymbol{\tau}_{3} \mid \eta_{1}=k\right\} P\left\{\eta_{1}=k\right\}
\end{aligned}
$$




$$
\begin{aligned}
& =\sum_{k=0}^{\infty} P\left\{\eta_{2}=m_{2}+k\right\} P\left\{\eta_{3}=m_{3}+k\right\} P\left\{\eta_{1}=k\right\} \\
& =\sum_{k=0}^{\infty} p_{\eta_{2}}\left(m_{2}+k\right) p_{\eta_{3}}\left(m_{3}+k\right) p_{\eta_{1}}(k) .
\end{aligned}
$$

Definition 2.2. We define as the two-dimensional discrete T-convolution the following operator of the three pmf $p_{\eta_{1}}(k), p_{\eta_{2}}(k), p_{\eta_{3}}(k)$ :

$$
\begin{aligned}
p_{\eta_{1}} \circledast p_{\eta_{2}} \circledast p_{\eta_{3}}\left(m_{1}, m_{2}, m_{3}\right) & \begin{cases}\sum_{k=0}^{\infty} p_{\eta_{1}}\left(m_{1}+k\right) p_{\eta_{2}}\left(m_{2}+k\right) p_{\eta_{3}}(k) & \text { if } \quad m_{1}>0, m_{2} \geq 0, \\
\sum_{k=0}^{\infty} p_{\eta_{2}}\left(m_{2}+k\right) p_{\eta_{3}}\left(m_{3}+k\right) p_{\eta_{1}}(k) & \text { if } \quad m_{2}>0, m_{3} \geq 0, \\
\sum_{k=0}^{\infty} p_{\eta_{3}}\left(m_{3}+k\right) p_{\eta_{1}}\left(m_{1}+k\right) p_{\eta_{2}}(k) & \text { if } \quad m_{1} \geq 0, m_{3}>0, \\
\sum_{k=0}^{\infty} p_{\eta_{3}}(k) p_{\eta_{2}}(k) p_{\eta_{1}}(k) & \text { if } \quad m_{1}=m_{2}=m_{3}=0 .\end{cases}
\end{aligned}
$$

\section{Generalized $n$-dimensional T-convolution}

As it is stated in [1], the whole of space can be filled with congruent rhombic dodecahedrons. We can sketch the basic procedure as follows. The whole of space can be filled with cubes, which are alternatively black and white to get a kind of threedimensional chessboard and then the black ones are removed. The empty space is decomposed into six pyramids that have square bases with a common vertex in the center of the empty cubic space. Now, a single white cube with a pyramid based on each of its faces forms a rhombic dodecahedron, it is obvious that these dodecahedrons can fill the whole of space, for instance, see Figure 157 on p. 152 of [1] or Figure 22.4c on p. 407 of [2]. Now, by knowing the probability distribution in the rhombic dodecahedron we can calculate the probability distribution in the rest of the space by using the polyhedron that fills the space.

Now, let us consider the filling of the space $\mathbb{R}^{n}$ with $n$-dimensional cubes having $t$ as their edge length. We will call adjacent cubes those cubes that have a common side. Let us choose one of these cubes and consider the Cartesian coordinate system with origin at the center of such a cube and coordinate axes passing through the centers of its opposite sides. In this coordinate system, each of the cubes filling the whole of space has coordinates divisible by $t$. All these cubes are classified into two classes. Cubes belonging to the first class have coordinates $\left(x_{1}, x_{2}, \ldots, x_{n}\right)$ for their centers and they satisfy $\left|x_{1}, x_{2}, \ldots, x_{n}\right| \vdots(2 t)$. The cubes that do not satisfy this condition belong to the 
second class. A cube of the first class is surrounded by $2 n$ adjacent cubes of the second class.

We can form an $n$-dimensional polyhedron $\mathcal{P}_{n}(t)$ by connecting the centers of every adjacent cube of the second class to the vertices of a common side of a cube of the first class. It is easily seen that $\mathbb{R}^{n}$ can be filled with such polyhedrons.

The number of vertices of a polyhedron $\mathcal{P}_{n}(t)$ equals $2^{n}+2 n$, this is the number of vertices of the first class plus the number of centers of adjacent cubes of the second class. The coordinates of these vertices are:

$$
\begin{aligned}
& \gamma_{0}\left(\frac{t}{2}, \frac{t}{2}, \ldots, \frac{t}{2}\right), \gamma_{1}\left(-\frac{t}{2}, \frac{t}{2}, \ldots, \frac{t}{2}\right), \gamma_{2}\left(\frac{t}{2},-\frac{t}{2}, \ldots, \frac{t}{2}\right), \gamma_{3}\left(-\frac{t}{2},-\frac{t}{2}, \ldots, \frac{t}{2}\right), \ldots, \\
& \gamma_{2^{n}-1}\left(-\frac{t}{2},-\frac{t}{2}, \ldots,-\frac{t}{2}\right), \varsigma_{1}(t, 0, \ldots, 0), \varsigma_{2}(0, t, \ldots, 0), \ldots, \varsigma_{n}(0,0, \ldots, t), \\
& \boldsymbol{\varsigma}_{n+1}(-t, 0, \ldots, 0), \varsigma_{n+2}(0,-t, \ldots, 0), \ldots, \varsigma_{2 n}(0,0, \ldots,-t),
\end{aligned}
$$

where $\gamma_{i}(\cdot), i=0, \ldots, 2^{n}-1$, are the vertices of a cube and $\varsigma_{j}(\cdot), j=1, \ldots, 2 n$, are the symmetry centers of the adjacent cubes.

Assume that $n=2 k, k \in \mathbb{N}$, and let us consider the vectors

$$
\varsigma_{1}(t, 0, \ldots, 0), \varsigma_{2}(0, t, \ldots, 0), \ldots, \varsigma_{n-1}(0,0, \ldots, t), \gamma_{2^{n-1}-1}\left(-\frac{t}{2}, \ldots,-\frac{t}{2}, \frac{t}{2}\right) .
$$

It is easy to verify, by using direct calculation, that the vectors are linearly independent and $\varsigma_{1}+\varsigma_{2}+\cdots+\varsigma_{n-1}+\gamma_{2^{n-1}-1}+\gamma_{2^{n-1}}=\mathbf{0}$. The magnitudes of these vectors are given by $\left|\varsigma_{1}\right|=\left|\varsigma_{2}\right|=\ldots=\left|\varsigma_{n-1}\right|=d$, and $\left|\gamma_{2^{n-1}-1}\right|=\left|\gamma_{2^{n}-1}\right|=\frac{\sqrt{n}}{2} t$. So, we can define the normalized vectors in the following manner:

$$
\boldsymbol{\tau}_{i}=\frac{\boldsymbol{\varsigma}_{i}}{\left|\boldsymbol{\varsigma}_{i}\right|}, i=1,2, \ldots, n-1, \quad \boldsymbol{\tau}_{n}=\frac{\gamma_{2^{n-1}-1}}{\left|\gamma_{2^{n-1}-1}\right|}, \quad \boldsymbol{\tau}_{n+1}=\frac{\gamma_{2^{n}-1}}{\left|\gamma_{2^{n}-1}\right|} .
$$

Similarly, when $n=2 k+1, k \in \mathbb{N}$, we can consider the vectors (vertices of the cube):

$$
\begin{array}{r}
\boldsymbol{\tau}_{1}^{*}(\underbrace{-\frac{t}{2},-\frac{t}{2}, \ldots,-\frac{t}{2}}_{k+1}, \frac{t}{2}, \frac{t}{2}, \ldots, \frac{t}{2}), \boldsymbol{\tau}_{2}^{*}(\frac{t}{2}, \underbrace{-\frac{t}{2},-\frac{t}{2}, \ldots,-\frac{t}{2}}_{k+1}, \frac{t}{2}, \frac{t}{2}, \ldots, \frac{t}{2}), \\
\ldots, \boldsymbol{\tau}_{n}^{*}(\frac{t}{2}, \frac{t}{2}, \ldots, \frac{t}{2}, \underbrace{\left.-\frac{t}{2},-\frac{t}{2}, \ldots,-\frac{t}{2}\right)}_{k+1}, \boldsymbol{\tau}_{n+1}^{*}\left(\frac{t}{2}, \frac{t}{2}, \frac{t}{2}, \ldots, \frac{t}{2}\right) .
\end{array}
$$

It can also be proved that these vectors are linearly independent, i.e. $\tau_{1}{ }^{*}+\tau_{2}{ }^{*}+\cdots+$ $\boldsymbol{\tau}_{n}^{*}+\boldsymbol{\tau}_{n+1}^{*}=\mathbf{0}$. They have modulus $\left|\boldsymbol{\tau}_{1}^{*}\right|=\left|\boldsymbol{\tau}_{2}^{*}\right|=\ldots=\left|\boldsymbol{\tau}_{n}^{*}\right|=\left|\boldsymbol{\tau}_{n+1}^{*}\right|=\frac{\sqrt{n}}{2} t$, then the normalized vectors are given by

$$
\boldsymbol{\tau}_{i}=\frac{\boldsymbol{\tau}_{i}^{*}}{\frac{\sqrt{n}}{2} t}, \quad i=1,2, \ldots, n, n+1
$$


Let $\xi_{i}, i=1,2, \ldots, n+1$, be independent random variables with pdf $f_{\xi_{i}}, i=$ $1,2, \ldots, n+1$, respectively. The pdf of the random vector $\boldsymbol{\xi}=\xi_{1} \boldsymbol{\tau}_{1}+\xi_{2} \boldsymbol{\tau}_{2}+\cdots+$ $\xi_{n+1} \tau_{n+1}$ in the set

$$
\left\{x_{1} \boldsymbol{\tau}_{1}+x_{2} \boldsymbol{\tau}_{2}+\cdots+x_{n} \boldsymbol{\tau}_{n} ; x_{1}, x_{2}, \ldots, x_{n} \geq 0\right\}
$$

is of the form

$$
f_{\boldsymbol{\xi}}\left(x_{1}, x_{2}, \ldots, x_{n}\right)=\frac{1}{V_{n}} \int_{-\infty}^{\infty} f_{\xi_{1}}\left(x_{1}+u\right) f_{\xi_{2}}\left(x_{2}+u\right) \cdots f_{\xi_{n}}\left(x_{n}+u\right) f_{\xi_{n+1}}(u) d u,
$$

where $V_{n}=\left|\left(\boldsymbol{\tau}_{1}, \boldsymbol{\tau}_{2}, \ldots, \boldsymbol{\tau}_{n}\right)\right|$ is the volume of the $n$-dimensional parallelepiped with edges $\tau_{1}, \tau_{2}, \ldots, \tau_{n}$.

Equation (3.1) can be deduced in a similar manner as equation (2.2). By following

Note 2:

Layout as on page 355 ? I.e.

"set $n$,

set $n-1$,

$\vdots$

set 1." the same approach as above we can obtain the pdf of the vector $\boldsymbol{\xi}$ in sets of the form

$$
\begin{aligned}
& \left\{x_{1} \boldsymbol{\tau}_{1}+x_{2} \boldsymbol{\tau}_{2}+\cdots+x_{n-1} \boldsymbol{\tau}_{n-1}+x_{n+1} \boldsymbol{\tau}_{n+1} ; x_{1}, x_{2}, \ldots, x_{n-1}, x_{n+1} \geq 0\right\}, \ldots, \\
& \left\{x_{2} \boldsymbol{\tau}_{2}+x_{3} \boldsymbol{\tau}_{3}+\cdots+x_{n} \boldsymbol{\tau}_{n}+x_{n+1} \boldsymbol{\tau}_{n+1} ; x_{2}, x_{3}, \ldots, x_{n}, x_{n+1} \geq 0\right\}
\end{aligned}
$$

Thus, by analogy to the three-dimensional case of the T-convolution in the plane we can define an $n$-dimensional T-convolution.

Definition 3.1. We define as the $n$-dimensional T-convolution the following operator of the $n+1$ pdf $f_{\xi_{1}}(x), f_{\xi_{2}}(x), \ldots, f_{\xi_{n+1}}(x)$ :

$$
\begin{aligned}
& f_{\xi_{1}} \circledast f_{\xi_{2}} \circledast \cdots \circledast f_{\xi_{n+1}}\left(x_{1}, x_{2}, \ldots, x_{n+1}\right) \\
& :=\left\{\begin{array}{r}
\frac{1}{V_{n}} \int_{-\infty}^{\infty} f_{\xi_{2}}\left(x_{2}+u\right) f_{\xi_{3}}\left(x_{3}+u\right) \cdots f_{\xi_{n+1}}\left(x_{n+1}+u\right) f_{\xi_{1}}(u) d u \\
\text { if } x_{2}>0, x_{3}, x_{4}, \ldots, x_{n+1} \geq 0, \\
\frac{1}{V_{n}} \int_{-\infty}^{\infty} f_{\xi_{1}}\left(x_{1}+u\right) f_{\xi_{3}}\left(x_{3}+u\right) \cdots f_{\xi_{n+1}}\left(x_{n+1}+u\right) f_{\xi_{2}}(u) d u \\
\text { if } x_{3}>0, x_{1}, x_{4}, x_{5}, \ldots, x_{n+1} \geq 0, \\
\vdots \\
\frac{1}{V_{n}} \int_{-\infty}^{\infty} f_{\xi_{1}}\left(x_{1}+u\right) f_{\xi_{2}}\left(x_{2}+u\right) \cdots f_{\xi_{n}}\left(x_{n}+u\right) f_{\xi_{n+1}}(u) d u \\
\text { if } x_{1}>0, x_{2}, x_{3}, \ldots, x_{n} \geq 0 .
\end{array}\right.
\end{aligned}
$$

Now, let us consider $n+1$ independent discrete random variables $\eta_{1}, \eta_{2}, \ldots, \eta_{n+1}$ with pmf

$$
p_{\eta_{1}}(k), p_{\eta_{2}}(k), \ldots, p_{\eta_{n+1}}(k), \quad k \in \mathbb{N}_{0}=\{0,1,2, \ldots, \infty\},
$$

respectively. The probability distribution of the random vector $\boldsymbol{\eta}=\eta_{1} \boldsymbol{\tau}_{1}+\eta_{2} \boldsymbol{\tau}_{2}+$ $\cdots+\eta_{n+1} \boldsymbol{\tau}_{n+1}$ on the lattice $\left\{m_{1} \boldsymbol{\tau}_{1}+m_{2} \boldsymbol{\tau}_{2}+\cdots+m_{n} \boldsymbol{\tau}_{n} ; m_{1}, m_{2}, \ldots, m_{n} \in \mathbb{N}_{0}\right\}$ 
is

$P\left\{\boldsymbol{\eta}=\left(m_{1} \boldsymbol{\tau}_{1}+m_{2} \boldsymbol{\tau}_{2}+\cdots+m_{n} \boldsymbol{\tau}_{n}\right)\right\}=\sum_{k=0}^{\infty} p_{\eta_{1}}\left(m_{1}+k\right) \cdots p_{\eta_{n}}\left(m_{n}+k\right) p_{\eta_{n+1}}(k)$.

Similarly, the corresponding distributions can be written for the lattices

$$
\begin{gathered}
\left\{m_{1} \boldsymbol{\tau}_{1}+m_{2} \boldsymbol{\tau}_{2}+\cdots+m_{n-1} \boldsymbol{\tau}_{n-1}+m_{n+1} \boldsymbol{\tau}_{n+1} ; m_{1}, m_{2}, \ldots, m_{n-1}, m_{n+1} \in \mathbb{N}_{0}\right\} \\
\left\{m_{1} \boldsymbol{\tau}_{1}+\cdots+m_{n-2} \boldsymbol{\tau}_{n-2}+m_{n} \boldsymbol{\tau}_{n}+m_{n+1} \boldsymbol{\tau}_{n+1} ; m_{1}, \ldots, m_{n-2}, m_{n}, m_{n+1} \in \mathbb{N}_{0}\right\} \\
\vdots \\
\left\{m_{2} \boldsymbol{\tau}_{2}+m_{3} \boldsymbol{\tau}_{3}+\cdots+m_{n} \boldsymbol{\tau}_{n}+m_{n+1} \boldsymbol{\tau}_{n+1} ; m_{2}, \ldots, m_{n}, m_{n+1} \in \mathbb{N}_{0}\right\} .
\end{gathered}
$$

Definition 3.2. We define as the $n$-dimensional discrete T-convolution the following operator of the $n+1$ pmf $p_{\eta_{1}}(k), p_{\eta_{2}}(k), \ldots, p_{\eta_{n+1}}(k)$ :

$$
\begin{aligned}
& p_{\eta_{1}} \circledast p_{\eta_{2}} \circledast \cdots \circledast p_{\eta_{n+1}}\left(m_{1}, m_{2}, \ldots, m_{n+1}\right) \\
& :=\left\{\begin{array}{c}
\sum_{k=0}^{\infty} p_{\eta_{2}}\left(m_{2}+k\right) p_{\eta_{3}}\left(m_{3}+k\right) \cdots p_{\eta_{n+1}}\left(m_{n+1}+k\right) p_{\eta_{1}}(k) \\
\text { if } m_{2}>0, m_{3}, m_{4}, \ldots, m_{n+1} \geq 0, \\
\sum_{k=0}^{\infty} p_{\eta_{1}}\left(m_{1}+k\right) p_{\eta_{3}}\left(m_{3}+k\right) \cdots p_{\eta_{n+1}}\left(m_{n+1}+k\right) p_{\eta_{2}}(k) \\
\quad \begin{array}{c}
\text { if } \quad m_{3}>0, m_{1}, m_{4}, m_{5}, \ldots, m_{n+1} \geq 0, \\
\vdots
\end{array} \\
\begin{array}{c}
\text { if } m_{1}>0, m_{2}, m_{3}, \ldots, m_{n} \geq 0, \\
\sum_{k=0}^{\infty} p_{\eta_{1}}\left(m_{1}+k\right) p_{\eta_{2}}\left(m_{2}+k\right) \cdots p_{\eta_{n}}\left(m_{n}+k\right) p_{\eta_{n+1}}(k) p_{\eta_{n}}(k) \cdots p_{\eta_{2}}(k) p_{\eta_{1}}(k)
\end{array} \\
\text { if } m_{1}=m_{2}=m_{3}=\ldots=m_{n+1}=0 .
\end{array}\right.
\end{aligned}
$$

\section{Application of T-convolution to the construction of $n$-dimensional distributions}

\subsection{A generalization of the Laplace distribution to the plane}

Let $\xi_{1}$ and $\xi_{2}$ be independent and exponentially distributed random variables with the same parameter $\lambda=1$, i.e. $f_{\xi_{1}}(x)=f_{\xi_{2}}(x)=e^{-x} \mathbf{I}_{\{x \geq 0\}}$. Then the random variable 
$\xi=\xi_{1}-\xi_{2}$ has the Laplace distribution,

$$
f_{\xi}(x)=\int_{-\infty}^{\infty} e^{-(x+u)} \mathbf{I}_{\{x+u \geq 0\}} e^{-u} \mathbf{I}_{\{u \geq 0\}} d u=\frac{1}{2} e^{-|x|}, \quad x \in \mathbb{R} .
$$

In a similar manner, let us suppose that $\xi_{1}, \xi_{2}, \xi_{3}$ are independent and exponentially distributed random variables with the same parameter $\lambda=1$. Then the random vector $\boldsymbol{\xi}=\xi_{1} \boldsymbol{\tau}_{1}+\xi_{2} \boldsymbol{\tau}_{2}+\xi_{3} \boldsymbol{\tau}_{3}$ has the distribution $f_{\boldsymbol{\xi}}=f_{\xi_{1}} \circledast f_{\xi_{2}} \circledast f_{\xi_{3}}(x, y, z)$, or equivalently,

$$
\begin{aligned}
& f_{\boldsymbol{\xi}}(x, y, z) \\
& =\left\{\begin{array}{c}
\frac{2}{\sqrt{3}} \int_{-\infty}^{\infty} e^{-(x+u)} \mathrm{I}_{\{x+u \geq 0\}} e^{-(y+u)} \mathrm{I}_{\{y+u \geq 0\}} e^{-u} \mathrm{I}_{\{u \geq 0\}} d u=\frac{2}{3 \sqrt{3}} e^{-(x+y)} \\
\text { for } x>0, y \geq 0 \\
\frac{2}{\sqrt{3}} \int_{-\infty}^{\infty} e^{-(y+u)} \mathrm{I}_{\{y+u \geq 0\}} e^{-(z+u)} \mathrm{I}_{\{z+u \geq 0\}} e^{-u} \mathrm{I}_{\{u \geq 0\}} d u=\frac{2}{3 \sqrt{3}} e^{-(y+z)} \\
\text { for } y>0, z \geq 0, \\
\frac{2}{\sqrt{3}} \int_{-\infty}^{\infty} e^{-(x+u)} \mathrm{I}_{\{x+u \geq 0\}} e^{-(z+u)} \mathrm{I}_{\{z+u \geq 0\}} e^{-u} \mathrm{I}_{\{u \geq 0\}} d u=\frac{2}{3 \sqrt{3}} e^{-(x+z)} \\
\text { for } x \geq 0, z>0 .
\end{array}\right.
\end{aligned}
$$

This distribution might be called the Laplace distribution in the plane. As usual, in constructing multidimensional distributions from well-known one-dimensional distributions, it is assumed that the marginal distributions are distributed like the given one-dimensional ones. We should notice that by intersecting the plot of the function $f_{\xi}(x, y, z)$ with a plane that is normal to its base and passing through the axis $\overrightarrow{O V}$ ( $O$ is the origin of vectors $\tau_{1}, \boldsymbol{\tau}_{2}, \boldsymbol{\tau}_{3}$ and $V$ is the vertex of the pdf), we will not obtain the one-dimensional Laplace distribution. However, if this plane is orthogonal to one of the axes $\boldsymbol{\tau}_{1}, \boldsymbol{\tau}_{2}, \boldsymbol{\tau}_{3}$, then the intersection is the function $h(s)=\frac{2}{3 \sqrt{3}} e^{-\sqrt{3}|s|}$ and $\frac{9}{4} h(s)$ is the Laplace distribution function. Hence, $f_{\boldsymbol{\xi}}(x, y, z)$ might be called the Laplace distribution in the plane. See the plot of this pdf in Figure 1, and its equal levels or contours in Figure 2.

\subsection{A generalization of the triangle distribution to the plane}

Suppose $\xi_{1}$ and $\xi_{2}$ are independent and uniformly distributed random variables on $[0,1]$. Then the random variable $\xi=\xi_{1}-\xi_{2}$ has the triangle distribution, namely

$$
f_{\xi}(x)=\int_{-\infty}^{\infty} \mathrm{I}_{[0,1]}(x+u) \mathrm{I}_{[0,1]}(u) d u=\left\{\begin{array}{ccc}
1-|x| & \text { if } & |x| \leq 1 \\
0 & \text { if } & |x|>1 .
\end{array}\right.
$$




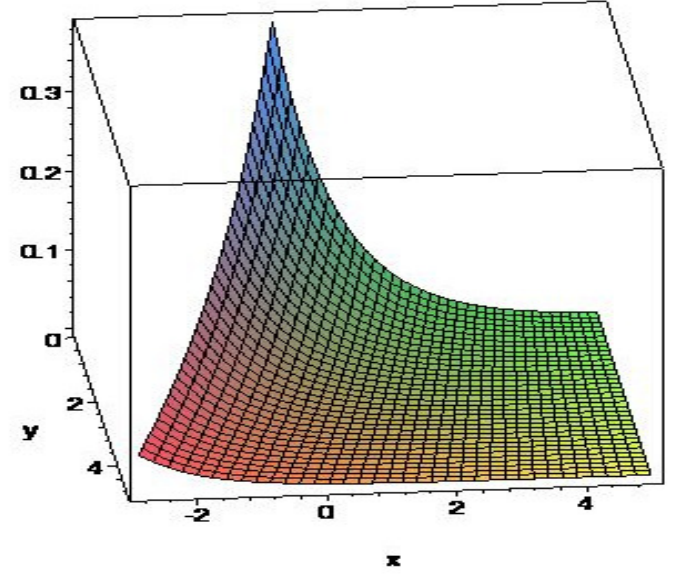

Figure 1. Laplace pdf in a $120^{\circ}$ sector of the plane.

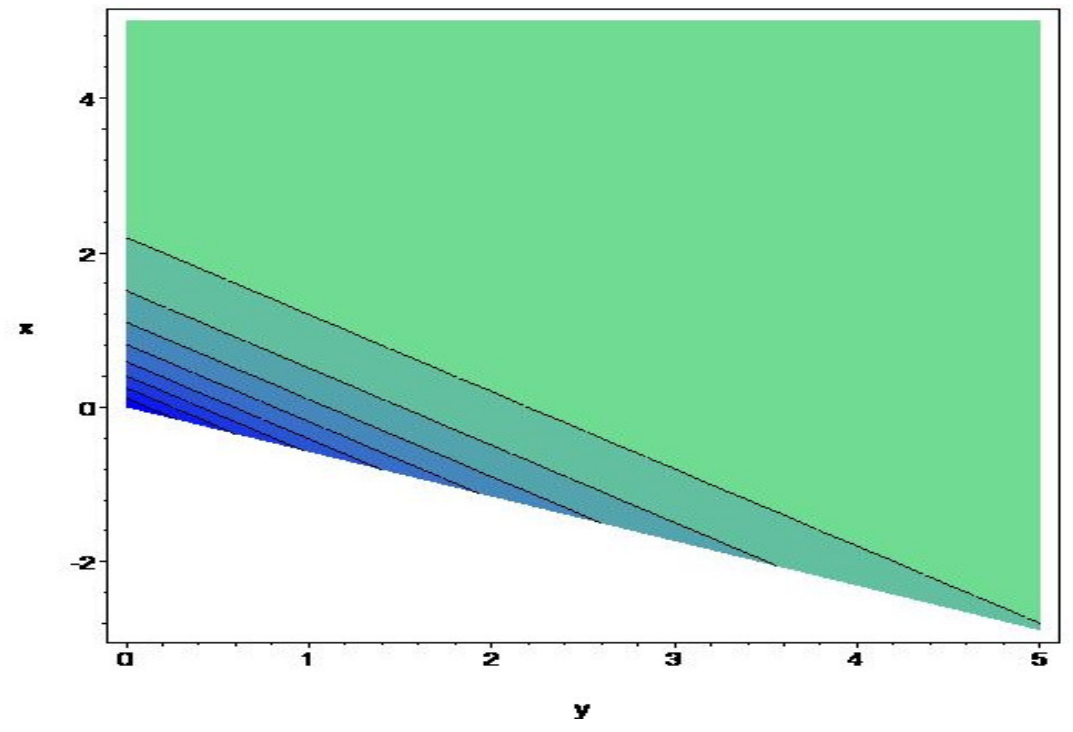

Figure 2. Contour of Laplace pdf in a $120^{\circ}$ sector of the plane. 
Now, we can generalize this result to the plane by considering the random vector $\boldsymbol{\xi}=\xi_{1} \boldsymbol{\tau}_{1}+\xi_{2} \boldsymbol{\tau}_{2}+\xi_{3} \boldsymbol{\tau}_{3}$ with pdf $f_{\boldsymbol{\xi}}\left(x_{1}, x_{2}, x_{3}\right)=f_{\xi_{1}} \circledast f_{\xi_{2}} \circledast f_{\xi_{3}}\left(x_{1}, x_{2}, x_{3}\right)$ given by

$$
f_{\boldsymbol{\xi}}\left(x_{1}, x_{2}, x_{3}\right)= \begin{cases}\frac{2}{\sqrt{3}}\left(1-\sup \left(x_{1}, x_{2}\right)\right) & \text { if } \quad 0<x_{1}<1,0 \leq x_{2} \leq 1, \\ \frac{2}{\sqrt{3}}\left(1-\sup \left(x_{2}, x_{3}\right)\right) & \text { if } \quad 0<x_{2}<1,0 \leq x_{3} \leq 1, \\ \frac{2}{\sqrt{3}}\left(1-\sup \left(x_{1}, x_{3}\right)\right) & \text { if } \quad 0<x_{3}<1,0 \leq x_{1} \leq 1,\end{cases}
$$

Note 3:

Do you mean: $0 \leq x_{3} \leq 1$ ?

Note 4:

Dito.

Note 5:

Please check sentence starting with "Intersections...". and it is equal to 0 when $x_{1}=x_{2}=x_{3}=0$ or $x_{1}=x_{2}=0,0 \leq x_{3} \leq 0$, and so on.

Consider a function $\|\cdot\|_{h}$ on vectors $\boldsymbol{x}=x_{1} \tau_{1}+x_{2} \tau_{2}+x_{3} \tau_{3}$ with ordinary addition and scalar multiplication operations. This function is defined by

$$
\left\|\left(x_{1}, x_{2}, x_{3}\right)\right\|_{h}= \begin{cases}\sup \left(x_{1}, x_{2}\right) & \text { if } 0<x_{1}<1,0 \leq x_{2} \leq 1 \\ \sup \left(x_{2}, x_{3}\right) & \text { if } 0<x_{2}<1,0 \leq x_{3} \leq 1 \\ \sup \left(x_{1}, x_{3}\right) & \text { if } 0<x_{3}<1,0 \leq x_{1} \leq 1\end{cases}
$$

and it is equal to 0 when $x_{1}=x_{2}=x_{3}=0$ or $x_{1}=x_{2}=0,0 \leq x_{3} \leq 0$, and so on.

It is easy to verify that $\|\cdot\|_{h}$ is a norm, and by using this notation we can write

$$
f_{\boldsymbol{\xi}}\left(x_{1}, x_{2}, x_{3}\right)=\frac{2}{\sqrt{3}}\left(1-\left\|\left(x_{1}, x_{2}, x_{3}\right)\right\|_{h}\right) .
$$

In this norm, $\|\left(x_{1}, x_{2}, x_{3} \|_{h}=r>0\right.$ represents a regular hexagon, so we might call it the hexagon norm. Hence, the pdf $f_{\boldsymbol{\xi}}\left(x_{1}, x_{2}, x_{3}\right)$ has a pyramid shape and it might be called the pyramid probability distribution. Intersections of this pyramid with three planes, where each of these planes passes through the center of the hexagon; also, it is perpendicular both to its base and to $\tau_{1}$ or $\tau_{2}$ or $\tau_{3}$. See the plot of this pdf in Figure 3, and its equal levels or contours in Figure 4. We only show one of the three sectors in the plane, each of $120^{\circ}$, and the pdf has a pyramid shape with a hexagon as its base.

\subsection{Discrete $n$-dimensional T-convolution of Poisson distributions}

Let us consider the $n$-dimensional discrete T-convolution of the $n+1$ independent Poisson distributed random variables $\eta_{1}, \eta_{2}, \ldots, \eta_{n+1}$ with parameters $\lambda_{1}, \lambda_{2}, \ldots$, $\lambda_{n+1}$, respectively. Each of these Poisson distributions has a pmf

$$
p_{\eta_{i}}\left(m_{i}\right)=\frac{\lambda_{i}^{m_{i}}}{m_{i} !} e^{-\lambda_{i}}, \quad i=1,2, \ldots, n+1 .
$$




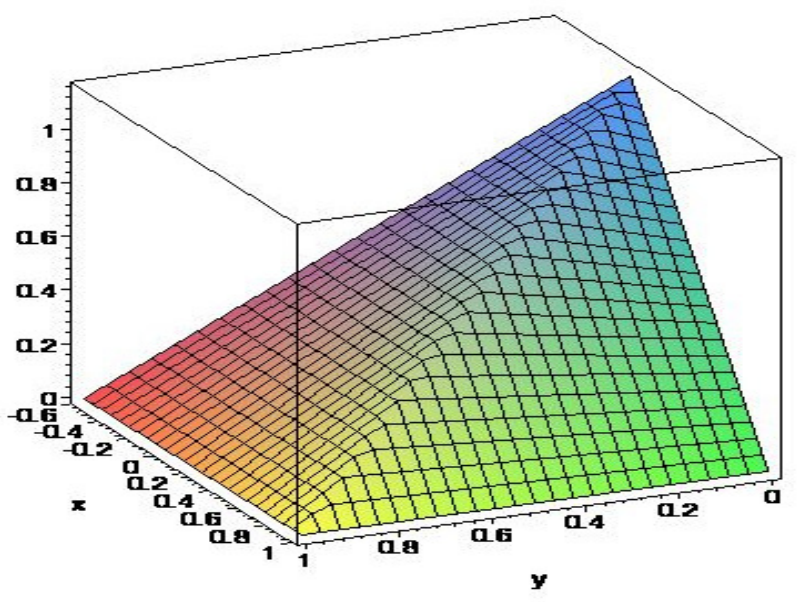

Figure 3. Triangle pdf in a $120^{\circ}$ sector of the plane (or pyramid pdf).

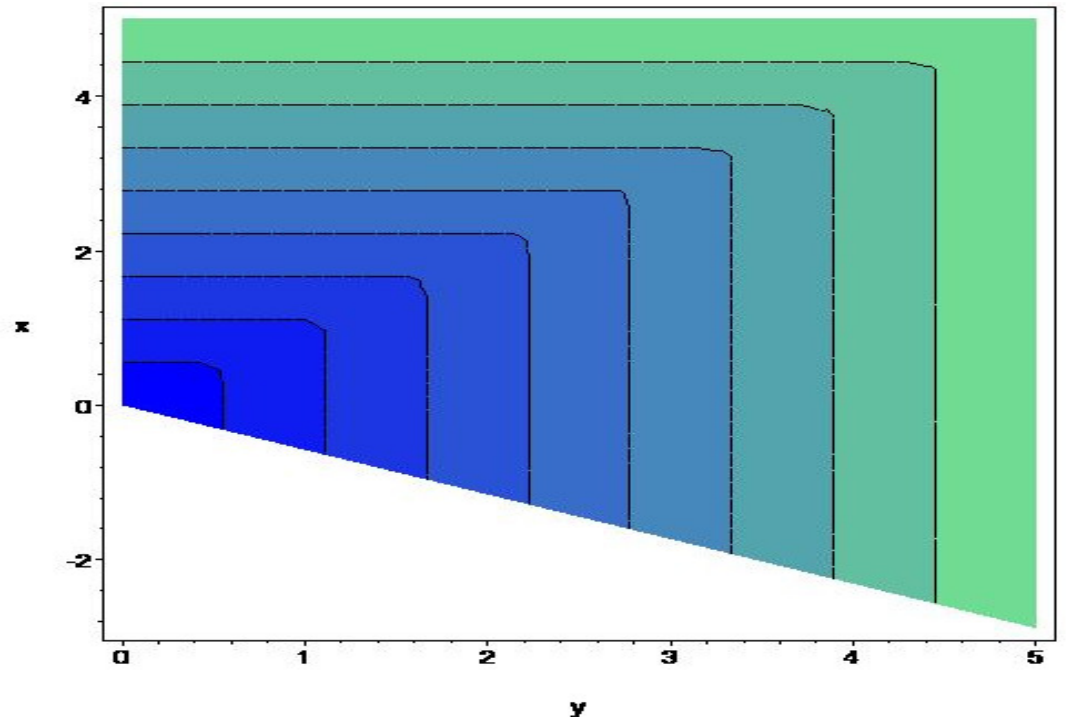

Figure 4. Contour of triangle pdf in a $120^{\circ}$ sector of the plane. 
Let us define the random vector $\boldsymbol{\eta}=\eta_{1} \boldsymbol{\tau}_{1}+\eta_{2} \boldsymbol{\tau}_{2}+\cdots+\eta_{n+1} \boldsymbol{\tau}_{n+1}$ on the lattice $\left\{m_{1} \boldsymbol{\tau}_{1}+m_{2} \boldsymbol{\tau}_{2}+\cdots+m_{n+1} \boldsymbol{\tau}_{n+1} ; m_{1}, m_{2}, \ldots, m_{n+1} \in \mathbb{N}_{0}\right\}$, then the T-convolution is given by

$$
p_{\boldsymbol{\eta}}\left(m_{1}, m_{2}, \ldots, m_{n+1}\right)=p_{\eta_{1}}\left(m_{1}\right) \circledast p_{\eta_{2}}\left(m_{2}\right) \circledast \cdots \circledast p_{\eta_{n+1}}\left(m_{n+1}\right),
$$

or equivalently,

$$
\begin{aligned}
p_{\boldsymbol{\eta}}\left(m_{1}, m_{2}, \ldots, m_{n+1}\right) & =\sum_{k=0}^{\infty} p_{\eta_{1}}\left(m_{1}+k\right) p_{\eta_{2}}\left(m_{2}+k\right) \cdots p_{\eta_{n}}\left(m_{n}+k\right) p_{\eta_{n+1}}(k) \\
& =\sum_{k=0}^{\infty}\left(\prod_{i=1}^{n} \frac{\lambda_{i}^{m_{i}+k}}{\left(m_{i}+k\right) !} e^{-\lambda_{i}}\right) \frac{\lambda_{n+1}^{k}}{k !} e^{-\lambda_{n+1}} \\
& =\prod_{i=1}^{n+1} e^{-\lambda_{i}} \sum_{k=0}^{\infty}\left(\prod_{i=1}^{n} \frac{\lambda_{i}^{m_{i}+k}}{\left(m_{i}+k\right) !}\right) \frac{\lambda_{n+1}^{k}}{k !} \\
& =\exp \left(-\sum_{i=1}^{n+1} \lambda_{i}\right) \sum_{k=0}^{\infty}\left(\frac{\prod_{i=1}^{n} \lambda_{i}^{m_{i}} \prod_{j=1}^{n} \lambda_{j}^{k}}{\prod_{i=1}^{n}\left(m_{i}+k\right) !}\right) \frac{\lambda_{n+1}^{k}}{k !} \\
& =\left(\prod_{i=1}^{n} \lambda_{i}^{m_{i}}\right) \exp \left(-\sum_{i=1}^{n+1} \lambda_{i}\right) \sum_{k=0}^{\infty}\left(\frac{\left(\prod_{j=1}^{n+1} \lambda_{j}\right)^{k}}{k ! \prod_{i=1}^{n}\left(m_{i}+k\right) !}\right) \\
& =M \sum_{k=0}^{\infty}\left(\frac{\left(\prod_{j=1}^{n+1} \lambda_{j}\right)^{k}}{k ! \prod_{i=1}^{n}\left(m_{i}+k\right) !}\right)
\end{aligned}
$$

where $M=\left(\prod_{i=1}^{n} \lambda_{i}^{m_{i}}\right) \exp \left(-\sum_{i=1}^{n+1} \lambda_{i}\right)$.

Furthermore, the pmf $p_{\boldsymbol{\eta}}\left(m_{1}, m_{2}, \ldots, m_{n+1}\right)$ can be written as

$M \sum_{k=0}^{\infty}(-1)^{k}\left(\frac{\left(\sqrt[n+1]{-\prod_{i=1}^{n+1} \lambda_{i}}\right)^{(n+1) k+\sum_{i=1}^{n}\left(m_{i}-1\right)}}{k ! \prod_{i=1}^{n}\left(\left(m_{i}-1\right)+k+1\right) !} \frac{1}{\left(\sqrt[n+1]{-\prod_{i=1}^{n+1} \lambda_{i}}\right)^{\sum_{i=1}^{n}\left(m_{i}-1\right)}}\right)$

or

$\frac{M}{\left(\sqrt[n+1]{-\prod_{i=1}^{n+1} \lambda_{i}}\right)^{\sum_{i=1}^{n}\left(m_{i}-1\right)}} \sum_{k=0}^{\infty}(-1)^{k}\left(\frac{\left(\sqrt[n+1]{-\prod_{i=1}^{n+1} \lambda_{i}}\right)^{(n+1) k+\sum_{i=1}^{n}\left(m_{i}-1\right)}}{k ! \prod_{i=1}^{n}\left(\left(m_{i}-1\right)+k+1\right) !}\right)$

Note 6:

Included:

"or" or

$$
\begin{aligned}
& p_{\boldsymbol{\eta}}\left(m_{1}, m_{2}, \ldots, m_{n+1}\right) \\
& =\frac{\left(\prod_{i=1}^{n} \lambda_{i}^{m_{i}}\right) \exp \left(-\sum_{i=1}^{n+1} \lambda_{i}\right)}{\left(\sqrt[n+1]{-\prod_{i=1}^{n+1} \lambda_{i}}\right)^{\sum_{i=1}^{n}\left(m_{i}-1\right)}} Z_{1,\left\langle\left(m_{1}-1\right),\left(m_{2}-1\right), \ldots,\left(m_{n}-1\right)\right\rangle}\left(\sqrt[n+1]{-\prod_{i=1}^{n+1} \lambda_{i}}\right)
\end{aligned}
$$




$$
=\left(e^{-\lambda_{n+1}} \prod_{i=1}^{n} \frac{\lambda_{i}^{m_{i}}}{m_{i} !} e^{-\lambda_{i}}\right) H_{\langle\rangle,\left\langle 1+m_{1}, 1+m_{2}, \ldots, 1+m_{n}\right\rangle}\left(\prod_{i=1}^{n+1} \lambda_{i}\right),
$$

where $Z_{1,\left\langle\left(m_{1}-1\right),\left(m_{2}-1\right), \ldots,\left(m_{n}-1\right)\right\rangle}$ is the Bessel type function of multi-index $\left\langle\left(m_{1}-1\right)\right.$, $\left.\left(m_{2}-1\right), \ldots,\left(m_{n}-1\right)\right\rangle$ [3], and $H_{\langle\rangle,\left\langle 1+m_{1}, 1+m_{2}, \ldots, 1+m_{n}\right\rangle}$ is the Barnes function [4].

\section{Application of T-convolution to the generalization of fading evolution to higher dimensions}

Suppose $\theta_{k} \geq 0, k \geq 0$, is a random variable distributed with pdf $f_{\theta_{k}}(t)=\frac{d}{d t} F_{\theta_{k}}(t)$. Consider the stochastic flow $\tau_{n}=\sum_{k=0}^{n} \theta_{k}$, and denote by $\{\xi(t), t>0\}$ the stochastic renewal process $\xi(t)=\max \left\{n: \tau_{n} \leq t\right\}$.

The process

$$
x(t)=\int_{0}^{t}(-a)^{\xi(s)} d s, \quad 0<a<1,
$$

is called the fading evolution in the random media $\xi(t)$.

Now, consider the random variable $\sigma$ defined by

$$
\sigma=\int_{0}^{\infty}(-a)^{\xi(s)} d s
$$

and denote by $F_{\sigma}(x)=P\{\sigma \leq x\}$ its cumulative distribution function (cdf).

It is easy to see that $F_{\sigma}(x)$ satisfies the integral equation

$$
F_{\sigma}(x)=\int_{0}^{\infty} f_{\theta_{k}}(t) F_{\sigma}\left\{\frac{x-u}{a^{2}}\right\} d u .
$$

The explicit solution of (5.1) when $\theta_{k}, k \geq 0$, are exponentially distributed was obtained in [5], and when $\theta_{k}, k \geq 0$, are 2-Erlang distributed was obtained in [6].

Now, we will generalize the fading evolution to the plane by using T-convolution. Denote by $\xi_{1}(t), \xi_{2}(t)$ and $\xi_{3}(t)$ three independent renewal processes, which are defined in a similar manner as the process $\xi(t)$. Let us define the three corresponding fading processes

$$
x_{1}(t)=\int_{0}^{t} a_{1}^{\xi_{1}(s)} d s, \quad x_{2}(t)=\int_{0}^{t} a_{2}^{\xi_{2}(s)} d s, \quad x_{3}(t)=\int_{0}^{t} a_{3}^{\xi_{3}(s)} d s,
$$

where $0<a_{k}<1, k=1,2,3$.

Let us define the vector process

$$
\boldsymbol{x}(t)=x_{1}(t) \boldsymbol{e}_{1}+x_{2}(t) \boldsymbol{e}_{2}+x_{3}(t) \boldsymbol{e}_{3},
$$

where $e_{1}, e_{2}, e_{3}$ are vectors for the T-convolution on the plane. Define

$$
\boldsymbol{\sigma}=\lim _{t \rightarrow \infty} x_{1}(t) \boldsymbol{e}_{1}+\lim _{t \rightarrow \infty} x_{2}(t) \boldsymbol{e}_{2}+\lim _{t \rightarrow \infty} x_{3}(t) \boldsymbol{e}_{3}=\sigma_{1} \boldsymbol{e}_{1}+\sigma_{2} \boldsymbol{e}_{2}+\sigma_{3} \boldsymbol{e}_{3},
$$


and denote by

$$
f_{\sigma_{k}}(x)=\frac{d}{d x} P\left\{\sigma_{k} \leq x\right\}, \quad k=1,2,3,
$$

the limiting distributions of the corresponding fading evolutions. Similarly, we can define the vector case $f_{\boldsymbol{\sigma}}(\boldsymbol{x})=\frac{d}{d \boldsymbol{x}} P\{\boldsymbol{\sigma} \leq \boldsymbol{x}\}$, where $\boldsymbol{x}=x_{1} \boldsymbol{e}_{1}+x_{2} \boldsymbol{e}_{2}+x_{3} \boldsymbol{e}_{3}$. Thus, it is easy to verify that the pdf $f_{\boldsymbol{\sigma}}(\boldsymbol{x})$ is the two-dimensional T-convolution corresponding to $f_{\sigma_{k}}(x)$, namely

$$
f_{\boldsymbol{\sigma}}\left(x_{1}, x_{2}, x_{3}\right)=f_{\sigma_{1}} \circledast f_{\sigma_{2}} \circledast f_{\sigma_{3}}\left(x_{1}, x_{2}, x_{3}\right) .
$$

By following similar derivations we can generalize the fading evolution to higher dimensions.

\section{Conclusions}

In this work we have introduced the definition of T-convolution and its application for the construction of symmetrical distributions in the plane and multivariate generalization of some well-known distributions. We think that T-convolutions might be also applied for modeling electromagnetic field intensities for some communication systems and it will be explored in our future investigation.

Acknowledgments. We thank the Tecnologico de Monterrey, Campus Monterrey, through the Research Chair in Telecommunications, for the support provided in the development of this work.

\section{References}

1. H. Coxeter, Introduction to Geometry, Second edition, John Wiley \& Sons, 1969.

2. H. Steinhaus, Mathematical Snapshots, Oxford University Press, 1950.

3. Y. D. Plotkin and A. F. Turbin, Higher order Bessel function equation, Asymptotic methods in the theory of random evolutions, National Ukrainian Academy of Sciences, Institute of Mathematics (1991), pp. 112-121 (Russian).

4. E. W. Barnes, The theory of the G function, Quart. J. Pure Appl. Math. 31 (1900), pp. 264-314.

5. I. V. Samoilenko, Fading Markov random evolution, Ukr. Math. J. 54(3) (2002), pp. 448459.

6. A. A. Pogorui, Stationary distributions of fading evolutions, Ukr. Math. J. 61(3) (2009), pp. 425-431. 
Received 2 April, 2009

\section{Author information}

A. A. Pogorui, Department of Mathematics, Zhytomyr State University, 10008, Ukraine. Email: pogor@zu.edu.ua

D. O. Kovalenko, Department of Mathematics, Zhytomyr State University, 10008, Ukraine. Email: ingrem@list.ru

Ramón M. Rodríguez-Dagnino, Tecnológico de Monterrey (ITESM), E. Garza Sada 2501 Sur, C.P. 64849, Monterrey, N.L., Mexico.

Email: rmrodrig@itesm.mx 